\title{
MOTIVACIÓN, GRUPO DE DEPORTE, NIVEL COMPETITIVO Y EDAD DEPORTIVA EN DEPORTISTAS CALDENSES ${ }^{1}$
}

\author{
MOTIVATION, GROUP SPORTS, COMPETITIVE LEVEL OF SPORTS AND AGE IN ATHLETES \\ FROM CALDAS
}

\author{
Héctor Haney Aguirre-Loaiza ${ }^{2}$ \\ Santiago Ramos Bermúdez ${ }^{3}$ \\ Adriana María Agudelo ${ }^{4}$
}

\section{Resumen}

Se estudian las diferencias entre los factores de motivación en relación con el género, grupos de deportes, edad deportiva y nivel competitivo en 205 deportistas caldenses $\left(M_{\text {edad }}=18,9, D E=5,2\right)$, que diligenciaron la Escala de Motivación Deportiva. Los 139 hombres y 66 mujeres, practicantes de 20 modalidades deportivas, fueron seleccionados a través de una muestra no probabilística en un diseño no experimental. Los datos demuestran que los deportistas puntuaron valores superior-medio en motivación extrínseca, motivación intrínseca por el conocimiento y la autosuperación, mientras en la amotivación y motivación integral del deportista la valoración es inferior. Las hipótesis de diferencias en el comportamiento de la motivación fueron rechazadas en variables de género, nivel competitivo y edad deportiva, sin que se encontraran promedios significativos. La variable grupo de deportes discriminó diferencias en motivación intrínseca por la autosuperación y motivación integral del deportista, favorable para los deportes de resistencia. Se concluye que los presentes datos apoyan la multidimensionalidad de la teoría de autodeterminación y el modelo jerárquico de la motivación. Se discute y se alienta nuevos resultados.

Palabras claves: motivación, motivación intrínseca, motivación, psicología del deporte, deportistas.

\section{Abstract}

The present study explores the differences between motivation factors related to gender, sports groups, sports age and competitive level in 205 athletes from Caldas (Medad= 18.9, SD = 5.2 ), which had been asked to answer the Sports Motivation Scale. The 139 men and 66 women, who practice 20 different sports were selected through a non-probabilistic sample in a non-experimental design. The data shows that athletes scored high- medium values in extrinsic motivation, intrinsic motivation by the knowledge and selfbetterment, in both, the discouragement and integral motivation of the athlete is lower. The hypothesis of differences in the behavior of the motivation variables were rejected in gender, age and sport competitive level in which no significant means were found. The variable that discriminated sports group differences in intrinsic motivation for self-improvement and comprehensive athlete motivation is favorable for endurance sports. We conclude that these data support the multidimensionality of self-determination theory and the hierarchical model of motivation. Discussed and new results are encouraged.

Keywords: motivation, extrinsic motivation, intrinsic motivation, sport psychology, athletes.

Fecha de recepción: 16 de octubre de 2014

Fecha de aprobación: 20 de marzo de 2015

Para citar este artículo:

Aguirre-Loaiza, H., Bermúdez, S. y Agudelo, A.M. (2015). Motivación, grupo de deporte, nivel competitivo y edad deportiva en deportistas Caldenses. Lúdica Pedagógica, (21), 141-151.

1 El presente trabajo hace parte del producto del programa beca-pasantía como Joven Investigador e Innovador por Colciencias en contrapartida con la Vicerrectoría de Investigaciones y Posgrados de la Universidad de Caldas con el código R-918-P-CC78.

2 Psicólogo. Licenciado en educación Física y Deportes. Docente Corporación Empresarial Universitaria Alexander von Humboldt. Docente Universidad del Quindío. Grupo de investigación Cumanday Actividad Física y Deporte. Correo electrónico: haney.aguirre@gmail.com

3 Magíster en Metodología del Entrenamiento Deportivo. Licenciado en Educación Física. Docente Universidad de Caldas. Departamento de Acción Física Humana. Grupo Cumanday Actividad Física y Deporte. Correo electrónico: santiago.ramos@ucaldas.edu.co

4 Magíster en Educación, Licenciada en Educación Básica con Énfasis en Educación Física, Recreación y Deportes, Investigadora Grupo Cumanday, Actividad Física y Deporte. Docente programa de Licenciatura en Educación Física y Deportes, Universidad del Quindío. Correo electrónico: adrimagugi@gmail.com 


\section{INTRODUCCIÓN}

La motivación es influyente en la competencia y el rendimiento para alcanzar los logros deportivos (Weinberg, 2009). Son diferentes los tipos de motivación que han servido de soporte para formular las perspectivas teóricas de la motivación (Lavallee et al., 2004) y por consiguiente, no se asume como un constructo unidimensional, sino que, por el contrario, es complejo y multidimensional. Así, actualmente se sobrepasan las líneas de estudios descriptivos a investigaciones con alcances a nivel inferencial con propósitos definidos de responder el por qué las personas practican un determinado deporte o lo abandonan.

Una de las propuestas en la arena científica es la teoría de Deci y Ryan (1985), quienes integraron tres conceptos: motivación intrínseca, extrínseca y amotivación. El compromiso con una actividad por el placer, la autonomía y la satisfacción de ejecutarla, se entiende como motivación intrínseca (Deci y Ryan, 1985; Dosil, 2008; Reeve, 2010). Por el lado de la motivación extrínseca, aquella depende por los refuerzos ambientales o externos para poder ejercer su práctica, (Dosil, 2008), y puntualmente está apoyada en la contingencias (Deci y Ryan, 1985). Finalmente, el concepto de amotivación se caracteriza por una manifestación de sentimientos de frustración, miedo o depresión, dado que el sujeto no expresa la intención de realizar alguna actividad y por tanto su actividad es desorganizada (Ryan y Deci, 2000).

La anterior propuesta recibe el nombre de la teoría de la autodeterminación (Deci y Ryan, 1985) y es una de las teorías de mayor producción investigativa dentro de la psicología de la actividad física y el deporte (Dosil, 2008). La teoría afirma que existe un continuo de autodeterminación entre dos polos o extremos, con la amotivación en el nivel mas bajo de la autodeterminación y la motivación intrínseca en el extremo más alto, y la motivación extrínseca en el centro. En este orden de ideas, se ha defendido que si las personas autorregulan sus conductas de manera voluntaria y volitiva, se favorecerá la implicación en la actividad, mientras que, por el contrario, hay un efecto controlador de factores ambientales (p. ej., el entrenador y/o padre de familia) actúan en contra de la autonomía y las percepciones autodeterminadas se verá frustrada la capacidad de elección de la persona, Ryan y Deci, 2000). La investigación actual recibe apoyo del continuo de la autodeterminación en investigaciones cuyo dominio se ha efectuado en contextos del deporte, el ejercicio físico y la actividad física (Chatzisarantis, Hagger, Biddle, Smith, y Wang, 2003), inclusive en poblaciones escolares mediadas por las clases de educación física (Standage, Duda, y Ntoumanis, 2005), al igual que reflexiones del estado del arte y alcances de la teoría de la autodeterminación han sido reportadas recientemente (González, Salguero, y Márquez, 2011).

Paralelamente, se registra como un aporte significativo la propuesta del modelo jerárquico de la motivación extrínseca e intrínseca (Vallerand, 1997 y 2000). Este modelo se apoya en la teoría de la autodeterminación (Ryan y Deci, 1985, 2000) y considera que la motivación se enmarca en determinantes y mediadores, los cuales se dividen en tres niveles: global, contextual y situacional (Dosil, 2008; Hernández, VasconcelosRaposo, Lazaro y Dosil, 2004). El nivel global es también conocido como personalidad-rasgo, que caracteriza a cada individuo y explica que unos deportistas tengan motivación extrínseca, otros, motivación intrínseca, y otros, amotivación. El nivel contextual, también conocido como vida cotidiana, se refiere a la orientación motivacional ante contextos específicos. El nivel situacional, entendido también como estado, hace referencia a que la motivación depende de un momento específico en el cual el deportista se encuentre (Dosil, 2008; Hernández et al., 2004).

A nivel metodológico e instrumental, son varios los desarrollos que se han establecido en la valoración de la motivación (Lavallee et al., 2004). En consonancia con el modelo jerárquico de la motivación y la teoría de la autodeterminación, una de las propuestas desarrolladas es la escala de motivación deportiva (EMD-SMS por su traducción al inglés Sport Motivation Scale), la cual se ha constituido en un instrumento de amplio alcance en la medición de la motivación en el amplio espectro del deporte. La EMD ha gozado de revisiones psicométricas que la han posicionado con un extenso reconocimiento y utilización en distintos contextos deportivos, con criterios de validez y confiabilidad. Algunos de los estudios que han evaluado las propiedades psicométricas de la EMD se han observado en deportistas norteamericanos (Martens y Webber, 2002), españoles (Balaguer, Castillo, y Duda, 2007; Guzmán, Carratalá, GarcíaFerriol y Carratalá, 2006; Núñez, Martín-Albo, y Navarro, 2007; Núñez, Martín-Albo, Navarro y González, 
2006) y en deportistas latinoamericanos (López, 2000; Martín-Albo et al., 2007; Rodríguez, y Lozada, 2007). La EMD ha integrado la motivación extrínseca, intrínseca y amotivación en siete factores, algunas revisiones mas actualizadas son el SMS-II (Pelletier, Rocchi, Vallerand, Deci y Ryan, 2013), han soportado seis factores afirmando que dicho instrumento adquiere valores satisfactorios a nivel del análisis factorial inclusive, concluyendo que la SMS-II se comporta mejor que la versión original y que otros instrumentos como Behavioral Regulation in Sport Questionnaire (BRSQ), argumentos que han sido refutados sin que se compruebe tal afirmación y se invita a nuevas investigaciones en este orden (Lonsdale et al., 2014).

Investigaciones sobre el constructo motivacional como objeto de estudio, tanto de la psicología del deporte como de las ciencias del deporte, han tenido impactos en diferentes planos, evidenciándose durante los últimos años una referencia amplia en la literatura del área, principalmente en deportistas cuyo contexto puede ser mas o menos próximo a las condiciones colombianas (Aguirre-Loaiza y González, 2014; Almagro, Sáenz-López, y Moreno-Murcia, 2012; Çetinkalp yTurksoy, 2011; García-Calvo et al. 2011; García-Mas et al., 2010; Guzmán-Lujan y Carratalá, 2006; Guzmán-Luján, García-Ferriol y Cervelló-Gimeno, 2005; López, Balaguer, Castillo y Tristán, 2011; López y Márquez, 2001; Núñez et al., 2011) y cuyas conclusiones han determinado la complejidad del constructo motivacional y la diversidad de variables personales y ambientales son objeto de estudio actualmente y se incentivan nuevas propuestas investigativas.

Estudiar la motivación en el deporte es importante en la medida que ayuda a comprender qué es lo que lleva, mantiene y eventualmente produce el retiro de las personas de la práctica deportiva (Dosil, 2008; Lavallee et al., 2004; Weinberg, 2009) con el fin de fundamentar las conductas adecuadas por parte de entrenadores, padres y dirigentes que incentiven la adherencia hacia las prácticas y hacer que estas respondan a la aplicación de estrategias en función de los deportistas, dado que al no contar con ellos, la materia prima, no hay clubes, ni participantes ni proceso alguno. Comprender la motivación es arrojar luz sobre una de las claves de los procesos de formación de deportistas (Durand,1988).

Otro de los aspectos importantes que subyace a la evaluación psicológica en el contexto del deporte, y principalmente lo relacionado con el estudio de la motivación, alude a las posibles inferencias de múltiples variables que pueden estar asociadas o ser predictoras con el comportamiento motivacional del deportista. Esto permitirá, a través de la investigación, tomar decisiones eficaces en programas oportunos y multidisciplinares soportados en un cuerpo de conocimiento científico. En este sentido, aunque se encuentra abundante literatura a nivel internacional y un desarrollo considerable en materia de las variables psicológicas relacionadas al deporte y la actividad física, en cuanto se refiere a Colombia, y principalmente al departamento Caldas, son escasos los aportes. En coherencia con lo anterior, y con respecto a lo expuesto en el modelo jerárquico de la motivación, se ha planteado como hipótesis las posibles diferencias entre los factores motivacionales en función de las variables de género, grupo de deportes, la edad deportiva y el nivel competitivo, por consiguiente, los objetivos planteados para la presente investigación fueron: (a) identificar los valores y puntajes de los factores motivacionales (motivación extrínseca, intrínseca y amotivación) en deportistas de los seleccionados caldenses y (b) establecer diferencias en los factores motivacionales en función a las variables tratadas.

\section{MÉTODO}

\section{Diseño}

Para cumplir los objetivos trazados, se efectuó un estudio no experimental de corte transversal (Kerlinger, 1988), debido a que no se efectuó ningún tipo de intervención o manipulación que desarrolle un principio de causalidad.

\section{Participantes}

A partir de una muestra no probabilística, participaron 205 deportistas que representan al departamento de Caldas, Colombia. La tabla 1 describe la distribución de acuerdo con las variables sociodemográficas. Con edades entre los 11 y 51 años de edad $(M=18,9$, $D E=5,2)$, de los cuales 139 fueron varones y 66 mujeres. El promedio de la edad deportiva fue de 7,9 $(D E=$ $4,7)$. Practicantes de veinte modalidades deportivas (ajedrez, baloncesto, bolos, boxeo, ciclismo, fútbol de salón, futsal, fútbol, gimnasia artística, hockey sobre patines, judo, karate-do, natación, actividades subacuáticas, patinaje, patinaje artístico, tenis de campo, 
triatlón, voleibol y wu-shu). Para efectos del análisis estadístico, y siguiendo la clasificación propuesta por Lanier (1997), dichos deportes fueron recogidos en cinco grupos: resistencia, pelota, combate, precisión y arte competitivo (PAC) y fuerza-velocidad.

Tabla 1. Características sociodemográficas de la población evaluada (frecuencia y porcentajes).

\begin{tabular}{|c|c|c|c|}
\hline Variable & $\begin{array}{c}\text { Todos } \\
\mathrm{n}=205(100 \%)\end{array}$ & $\begin{array}{c}\text { Varones } \\
\mathrm{n}=139(68 \%)\end{array}$ & $\begin{array}{c}\text { Mujeres } \\
n=66(32 \%)\end{array}$ \\
\hline Edad & n $(\%)$ & n $(\%)$ & n $(\%)$ \\
\hline $11-24$ & $178(86.8)$ & $121(87,1)$ & $57(36,4)$ \\
\hline $25-37$ & $26(12,7)$ & $17(12,2)$ & $9(13,7)$ \\
\hline $38-51$ & $1(0,5)$ & $1(0,7)$ & $0(0,0)$ \\
\hline \multicolumn{4}{|l|}{ Escolaridad } \\
\hline Primaria & $2(0,97)$ & $0(0.0)$ & $2(3.0)$ \\
\hline Secundaria & $122(59.5)$ & $90(64.7)$ & $33(50,0)$ \\
\hline Superior & $76(37,07)$ & $45(32,4)$ & $31(47,0)$ \\
\hline Posgrado & $4(1,95)$ & $4(2,9)$ & $0(0,0)$ \\
\hline \multicolumn{4}{|c|}{ Estrato socioeconómico } \\
\hline 1-2 (bajo) & $56(27,3)$ & $36(25,9)$ & $20(30,3)$ \\
\hline 3-4 (medio) & $113(55,1)$ & $77(55,4)$ & $36(54,4)$ \\
\hline 5-6 (alto) & $33(16,1)$ & $23(16,5)$ & $10(15,2)$ \\
\hline Datos perdidos & $3(1,5)$ & $3(2,2)$ & $0(0,0)$ \\
\hline \multicolumn{4}{|c|}{ Lugar de nacimiento } \\
\hline Manizales & $167(81,5)$ & $113(55,1)$ & $54(26,3)$ \\
\hline Caldas & $10(4,9)$ & $6(2,9)$ & $4(2,0)$ \\
\hline Colombia & $27(13,2)$ & $20(9,8)$ & $7(3,4)$ \\
\hline Otro país & $1(0,5)$ & $0(0,0)$ & $1(0,5)$ \\
\hline
\end{tabular}

\section{Técnicas e instrumentos}

El instrumento aplicado para evaluar la motivación fue la escala de motivación deportiva (EMD) desarrollada por Briere, Vallerand, Blais y Pelletier (1995), traducida al castellano por López (2000), quien obtuvo un coeficiente alfa de Cronbach de $\alpha=0,89$. Esta escala permite evaluar la motivación extrínseca, la motivación intrínseca (al conocimiento, estética, autosuperación y al cumplimiento), la amotivación y motivación integral deportiva. La EMD original tiene 28 ítems, no obstante la versión de López (2000) tiene un ítem adicional, para un total de 29. Las respuestas se puntúan en una escala Lickert que oscilaba entre 1 (totalmente en desacuerdo) y 7 (totalmente de acuerdo) ante la pregunta ¿por qué práctica deporte? La versión empleada presenta un total de siete factores: factor 1 motivación extrínseca, factor 2 motivación intrínseca al conocimiento, factor 3 motivación intrínseca a la estética, factor 4 motivación intrínseca a la autosuperación, factor 5 motivación intrínseca al cumplimiento, factor 6 amotivación y factor 7 motivación integral-deportiva. La variables sociales y demográficas, el nivel competitivo y la edad deportiva fueron establecidos por un autoinforme a través de un cuestionario ad hoc elaborado por los investigadores. Los valores asignados en la puntuación normalizada se expresan en percentiles los cuales corresponde a: (Pc $\leq 5$ Muy inferior, $\mathrm{Pc} \leq 25$ Inferior, $\mathrm{Pc} \leq 30$ Medio, $\mathrm{Pc} \leq 75$ Superior, y Pc $\geq 95$ Muy Superior).

\section{ANÁLISIS DE DATOS}

Los datos fueron digitados en una base en Excel y tratados con el programa SPSS. Fueron calculadas medidas de tendencia central (media) y dispersión (desviación típica). Para establecer la normalidad de la distribución 
de los datos se empleó la prueba de Kolmogorov-Smirnov ( $p>.05)$, la cual encontró que los datos no se ajustaban al la distribución normal de los datos, por consiguiente se empleó la prueba no paramétrica de MannWhitney en dos muestras independientes. Para más de dos muestras, las diferencias fueron contrastadas a través de la prueba de Kruskal-Wallis. Cumpliendo las disposiciones vigentes, los deportistas firmaron un consentimiento informado y en concordancia a la ley 1090 y las condiciones pertinentes de la ley del psicólogo.

\section{RESULTADOS}

Los puntajes promedios, desviaciones estándar y puntajes percentiles se describen en la tabla 2 . En esencia, y siguiendo los baremos propuestos por López (2000), se encontró que los deportistas caldenses puntuaron en los factores 1, 2 y 4 (motivación extrínseca, motivación intrínseca por conocimiento, motivación intrínseca por autosuperación) valores de superior-medio. En tanto los factores 3 y 5 (motivación intrínseca por estética, y motivación intrínseca por cumplimiento) se valoran como medio ( $P c=50$ y en los factores VI y VII (amotivación, motivación integral del deportista) la categoría inferior.

Tabla 2. Valores de los factores motivacionales en los deportistas caldenses.

\begin{tabular}{|l|c|c|c|c|c|c|c|}
\hline \multirow{2}{*}{ Medidas } & \multicolumn{7}{|c|}{ Factores motivacionales } \\
\cline { 2 - 9 } & F-1 & F-2 & F-3 & F-4 & F-5 & F-6 & F-7 \\
\hline Media (DE) & $27.2(8.7)$ & $23.3(4.5)$ & $21.9(4.9)$ & $25.2(3.0)$ & $25.6(3.1)$ & $31.4(4.3)$ & $11.9(2.2)$ \\
\hline Percentil & 75 & 75 & 50 & 75 & 50 & 10 & 25 \\
\hline Valores & $\begin{array}{c}\text { Superior/ } \\
\text { medio }\end{array}$ & $\begin{array}{c}\text { Superior/ } \\
\text { medio }\end{array}$ & Medio & $\begin{array}{c}\text { Superior/ } \\
\text { medio }\end{array}$ & Medio & Inferior & Inferior \\
\hline
\end{tabular}

Nota: F-1= motivación extrínseca, F-2 = motivación intrínseca por conocimiento, F-3 = motivación intrínseca por estética, F-4 = motivación intrínseca por autosuperación, F-5 = motivación intrínseca por cumplimiento, F-6 = amotivación y F-7 = motivación integral del deportista.

\section{Género}

En cuanto al género, se presentaron promedios no significativos en los distintos factores de la motivación.
Tal y como se aprecia en la tabla 3, se describen las medias y desviaciones estándar de los factores motivacionales en comparación al género.

Tabla 3. Factores de la motivación en función al género.

\begin{tabular}{|c|c|c|c|c|c|}
\hline \multirow{3}{*}{ Factor } & \multicolumn{2}{|c|}{ Género } & \multirow{2}{*}{$\begin{array}{c}\text { Todos } \\
(n=205)\end{array}$} & \multicolumn{2}{|c|}{ Mann-Whitney } \\
\hline & $\begin{array}{c}\text { Hombres } \\
(n=139)\end{array}$ & $\begin{array}{l}\text { Mujeres } \\
(n=66)\end{array}$ & & \multirow[t]{2}{*}{$\mathrm{U}$} & \multirow{2}{*}{$\mathrm{p}$} \\
\hline & $\mathrm{M}(\mathrm{DE})$ & M (DE) & $\mathrm{M}(\mathrm{DE})$ & & \\
\hline F1 & $27,6(8,7)$ & $26,3(9,0)$ & $27,2(8,8)$ & 4142,5 & ,262 \\
\hline F2 & $23,6(4,4)$ & $22,8(4,8)$ & $23,3(4,6)$ & 4153,5 & 272 \\
\hline F3 & $21,9(4,8)$ & $21,9(5,4)$ & $21,9(5,0)$ & 4419,5 & ,672 \\
\hline F4 & $25,3(2,8)$ & $24,8(3,5)$ & $25,2(3,0)$ & 4330,5 & ,511 \\
\hline F5 & $25,9(2,7)$ & $25,0(3,9)$ & $25,6(3,2)$ & 4125,0 & 232 \\
\hline F6 & $31,6(4,0)$ & $30,9(5,0)$ & $31,4(4,3)$ & 4089,0 & 198 \\
\hline F7 & $11,8(2,3)$ & $12,1(2,1)$ & $11,9(2,2)$ & 4251,5 & 387 \\
\hline
\end{tabular}

Nota: $\mathrm{M}=$ Media, DE = Desviación Estándar, Factores motivacionales: $\mathrm{F}-1$ = motivación extrínseca, F-2 = motivación intrínseca por conocimiento, F-3 = motivación intrínseca por estética, F-4 = motivación intrínseca por autosuperación, F-5 = motivación intrínseca por cumplimiento, F-6 = amotivación y F-7 = motivación integral del deportista. 
El contraste de los puntajes motivacionales analizados entre los varones y las mujeres no arrojó diferencias significativas. La descripción de las medias identifica que los hombres lograron mejores puntuaciones en los factores 1, 2, 4 y 6 (motivación extrínseca, motivación integral al conocimiento y a la autosuperación, y amotivación); mientras que las mujeres puntuaron mejor promedio en el factor 7 (motivación integral). Para los factores 3 y 5 los puntajes fueron similares entre ambos géneros. Por su parte, el factor de amotivación caracterizó los valores más altos observados en ambos géneros.

\section{Grupos de deportes}

Por grupos de deporte, los atletas observados según la frecuencia de mayor a menor correspondieron en el siguiente orden de deportes: pelota, combate, PAC, fuerza-velocidad y, finalmente, resistencia. En el contraste de las medias entre los grupos de deportes y los factores motivacionales, únicamente se destacan diferencias significativas en el factor 4 y 7 (motivación intrínseca a la autosuperación y motivación integral), en los demás factores no se encontraron promedios significativos.

Tabla 4. Factores de la motivación en función a los grupos de deportes.

\begin{tabular}{|c|c|c|c|c|c|c|c|c|}
\hline \multirow{4}{*}{ Factores } & \multicolumn{6}{|c|}{ Grupos de deportes } & \multirow{2}{*}{\multicolumn{2}{|c|}{ Kruskal-Wallis }} \\
\hline & $\begin{array}{l}\text { Fuerza- } \\
\text { Velocidad }\end{array}$ & Resistencia & Pelota & Combate & PAC & Todos & & \\
\hline & $\begin{array}{c}n=19 \\
(9,3 \%)\end{array}$ & $\begin{array}{c}n=18 \\
(8,8 \%)\end{array}$ & $\begin{array}{c}n=115 \\
(56,1 \%)\end{array}$ & $\begin{array}{c}n=29 \\
(14,1 \%)\end{array}$ & $\begin{array}{c}n=24 \\
(11,7 \%)\end{array}$ & $\begin{array}{c}n=205 \\
(100 \%)\end{array}$ & \multirow{2}{*}{$\begin{array}{c}\chi^{2} \\
(\mathrm{gl}, 4)\end{array}$} & \multirow{2}{*}{$p$} \\
\hline & $M(D E)$ & $M(D E)$ & $M(D E)$ & $M(D E)$ & $M(D E)$ & $M(D E)$ & & \\
\hline F1 & $25,8(9,7)$ & $30,7(7,6)$ & $26,4(8,7)$ & $28,8(8,8)$ & $27,8(8,8)$ & $27,2(8,7)$ & 5,17 & ,270 \\
\hline F2 & $22,6(5,1)$ & $24,5(4,0)$ & $23,5(4,2)$ & $21,8(5,1)$ & $24,6(5,1)$ & $23,3(4,5)$ & 8,93 & ,063 \\
\hline F3 & $22,3(4,7)$ & $23,2(3,6)$ & $21,7(5,1)$ & $21,3(6,0)$ & $22,8(4,5)$ & $21,9(4,9)$ & 1,60 & ,809 \\
\hline $\mathrm{F} 4$ & $25,8(4,3)$ & $26,1(2,1)$ & $24,8(3,0)$ & $25,3(2,6)$ & $26,3(3,0)$ & $25,2(3,0)$ & 15,66 & ,004 \\
\hline F5 & $24,8(4,2)$ & $26,4(1,8)$ & $25,6(2,9)$ & $25,4(3,3)$ & $26,0(3,9)$ & $25,6(3,1)$ & 3,52 & ,474 \\
\hline F6 & $30,7(5,1)$ & $30,0(4,2)$ & $31,7(4,1)$ & $32,0(5,0)$ & $31,0(3,8)$ & $31,4(4,3)$ & 6,20 & 185 \\
\hline F7 & $11,3(2,3)$ & $12,9(1,4)$ & $11,8(2,3)$ & $11,4(2,3)$ & $12,8(2,0)$ & $11,9(2,2)$ & 14,12 & ,007 \\
\hline
\end{tabular}

Nota: $N=205, M=$ Media, $D E=$ Desviación Estándar. Fza-vel= Fuerza y Velocidad. Resist= Resistencia, PAC= Precisión y arte competitivo. F-1= motivación extrínseca, F-2 = motivación intrínseca por conocimiento, F-3 = motivación intrínseca por estética, F-4 = motivación intrínseca por autosuperación, F-5 = motivación intrínseca por cumplimiento, F- $6=$ amotivación y F-7 = motivación integral del deportista.

Los factores motivacionales en función al grupo de deporte demostraron diferencias significativas en el factor 4 , motivación intrínseca por la autosuperación, $\chi^{2}(4, N=205)=15.6, p=.004$; y en el factor 7 , motivación integral del deportista, $\chi^{2}(4, N=205)=14.2, p=.007$. En ambos factores diferenciadores, se encontraron promedios que son favorables para los deportes de resistencia y de PAC.

describen medias superiores al promedio general en los factores 2, 4, 5 y 7 (motivación intrínseca: al conocimiento, a la autosuperación y al cumplimiento; y motivación integral-deportiva).

En términos generales, cabe destacar que los puntajes observados en los deportes de resistencia, en comparación con el promedio general y de los demás grupos de deporte, se perfilan con las mayores medias en todos los factores, además se observó que el factor 6 (amotivación) corresponde al puntaje más bajo, es decir, son los deportistas que menos amotivación enfrentan ante la práctica deportiva. Asimismo, se destacan los puntajes de los deportes de PAC, que siguen la misma línea de comportamiento de los deportes de resistencia; se

Entre tanto, los deportes de combate presentan el puntaje más alto en amotivación $(M=32,0, D E=5,0)$, conjuntamente a este grupo, los deportes de fuerza y velocidad arrojaron los promedios más bajos en los factores 2, 3 y 4 (motivación intrínseca al conocimiento, a la estética, y a la autosuperación). Finalmente, frente a los deportes de pelota, se puede decir que obtienen datos que se ajustan al promedio general sin diferencias estadísticamente significativas. 


\section{Nivel competitivo}

La descripción del nivel de competencia en relación con los factores se estableció en cuatro niveles: regional, nacional, continental y mundial. La tabla 5 describe los respectivos puntajes. De la muestra estudiada, $n=37$ casos no reportaron la información pertinente al nivel competitivo. El máximo nivel competitivo corresponde a una variable que puede señalar algún efecto o asociación con la motivación del deportista. Si bien no se encontraron diferencias significativas entre el nivel competitivo y los factores motivacionales, parece existir un dominio en los puntajes arrojados por los deportistas que se encuentran en el nivel mundial.

Tabla 5. Factores de la motivación y el nivel competitivo.

\begin{tabular}{|c|c|c|c|c|c|c|}
\hline \multirow{4}{*}{ Factores } & \multicolumn{4}{|c|}{ Nivel competitivo } & \multirow{2}{*}{\multicolumn{2}{|c|}{ Kruskal-Wallis }} \\
\hline & \multirow{3}{*}{$\begin{array}{c}\text { Regional } \\
\qquad n=62 \\
M(D E)\end{array}$} & \multirow{3}{*}{$\begin{array}{c}\text { Nacional } \\
n=82 \\
\text { M (DE) }\end{array}$} & \multirow{3}{*}{$\begin{array}{l}\text { Continental } \\
\qquad n=16 \\
\mathrm{M}(\mathrm{DE})\end{array}$} & \multirow{3}{*}{$\begin{array}{c}\text { Mundial } \\
n=8 \\
M(D E)\end{array}$} & & \\
\hline & & & & & $\chi^{2}$ & \\
\hline & & & & & (gl. 3) & $p$ \\
\hline $\mathrm{F} 1$ & $27,4(9,5)$ & $26,8(8,7)$ & $26,4(7,2)$ & $28,3(9,3)$ & ,659 & 883 \\
\hline F2 & $23,6(4,9)$ & $23,0(4,4)$ & $23,4(4,2)$ & $23,5(4,0)$ & 1,825 & ,610 \\
\hline F3 & $21,4(5,2)$ & $21,7(5,0)$ & $24,4(3,4)$ & $24,5(4,4)$ & 7,205 & ,066 \\
\hline $\mathrm{F} 4$ & $24,7(3,6)$ & $25,2(3,0)$ & $26,6(1,3)$ & $26,5(1,7)$ & 4,190 & ,242 \\
\hline F5 & $25,6(3,4)$ & $25,3(3,1)$ & $26,1(2,1)$ & $27,0(1,7)$ & 3,710 & ,294 \\
\hline F6 & $31,6(3,9)$ & $31,4(4,6)$ & $30,3(4,6)$ & $\mathbf{3 4 , 5}(0,9)$ & 7,597 & ,055 \\
\hline F7 & $11,9(2,4)$ & $11,7(2,2)$ & $13,1(0,8)$ & $12,9(1,8)$ & 6,882 & ,076 \\
\hline
\end{tabular}

Nota: $n=168$. Factores motivacionales: F-1= motivación extrínseca, F-2 = motivación intrínseca por conocimiento, F-3 = motivación intrínseca por estética, F-4 = motivación intrínseca por autosuperación, F-5 = motivación intrínseca por cumplimiento, F-6 = amotivación y F-7 = motivación integral del deportista.

\section{Edad deportiva}

En función a la variable de edad deportiva, categorizada por cinco rangos de intervalo y expresados en la tabla 6. Sin que se hubieran establecido diferencias estadísticamente significativas en los promedios, se observó que el grupo de los menos experimentados arrojaron promedio superiores en comparación con los demás grupos en factores como 1, 2, 5 y 7. De igual modo, cabe destacar que los deportistas que tienen más años en edad deportiva no arrojaron promedios superiores comparados con los demás grupos con menor experiencia

Tabla 6. Factores de la motivación y el nivel competitivo.

\begin{tabular}{|c|c|c|c|c|c|c|c|}
\hline \multirow{4}{*}{ Factores } & \multicolumn{5}{|c|}{ Edad deportiva (años) } & \multirow{2}{*}{\multicolumn{2}{|c|}{ Kruskal-Wallis }} \\
\hline & \multirow{2}{*}{$\begin{array}{l}0 \text { a } 4 \\
n=19\end{array}$} & \multirow{2}{*}{$\begin{array}{l}5 \text { a } 9 \\
n=75\end{array}$} & \multirow{2}{*}{$\begin{array}{c}10 \text { a } 14 \\
n=56\end{array}$} & \multirow{2}{*}{$\begin{array}{c}15 \text { a } 19 \\
n=10\end{array}$} & \multirow{2}{*}{$\begin{array}{c}\text { Más de } 20 \\
n=5\end{array}$} & & \\
\hline & & & & & & & \\
\hline & $\mathrm{M}(\mathrm{DE})$ & $\mathrm{M}(\mathrm{DE})$ & $\mathrm{M}(\mathrm{DE})$ & $\mathrm{M}(\mathrm{DE})$ & $\mathrm{M}(\mathrm{DE})$ & (gl t & $P$ \\
\hline $\mathrm{F} 1$ & $28,9(8,4)$ & $25,9(9,2)$ & $28,4(8,15)$ & $22,8(10,0)$ & $24,2(7,5)$ & 6,94 & ,225 \\
\hline $\mathrm{F} 2$ & $23,8(4,8)$ & $23,3(4,7)$ & $23,2(3,98)$ & $22,1(6,1)$ & $22,5(2,0)$ & 5,54 & ,354 \\
\hline F3 & $21,6(5,1)$ & $21,7(5,4)$ & $22,2(4,35)$ & $22,4(5,8)$ & $20,0(4,5)$ & 3,62 & 604 \\
\hline $\mathrm{F} 4$ & $25,1(2,6)$ & $25,4(2,8)$ & $25,1(3,3)$ & $23,9(4,3)$ & $25,2(2,2)$ & 3,31 & ,652 \\
\hline F5 & $26,1(2,4)$ & $25,3(3,5)$ & $25,8(3,1)$ & $25,7(2,2)$ & $22,5(0,5)$ & 8,89 & 114 \\
\hline F6 & $31,0(5,1)$ & $\mathbf{3 1 , 8}(4,2)$ & $31,7(3,5)$ & $29,3(4,5)$ & $27,5(6,1)$ & 6,90 & 228 \\
\hline F7 & $12,3(2,1)$ & $11,6(2,3)$ & $11,9(2,2)$ & $11,5(1,9)$ & $11,5(2,0)$ & 6,47 & 262 \\
\hline
\end{tabular}

Nota: $n=195$. Factores motivacionales: F-1= motivación extrínseca, F-2 = motivación intrínseca por conocimiento, F-3 = motivación intrínseca por estética, F-4 = motivación intrínseca por autosuperación, F-5 = motivación intrínseca por cumplimiento, F-6 = amotivación y F-7 = motivación integral del deportista. 


\section{DISCUSIÓN}

Los objetivos del presente estudio correspondieron a identificar las diferencias entre el constructo de la motivación y variables en función al género, grupo de deporte, nivel competitivo y edad deportiva. Solo se encontraron diferencias en el comportamiento de los factores de motivación intrínseca a la autosuperación y motivación integral (factores 4 y 7) según las variables del grupo de deporte. Por otro lado, las variables restantes, es decir, género, edad deportiva y nivel competitivo, no discriminaron promedios en los factores motivacionales. En este sentido, las hipótesis planteadas por el grupo investigador son rechazadas y, por tanto, se asume que no hay diferencias en lo que respecta al comportamiento motivacional en el género, el nivel competitivo (regional, nacional, continental o mundial) y la edad deportiva de los participantes caldenses. De acuerdo con esto, los anteriores hallazgos del presente estudio permiten acotar algunos aspectos de importancia.

La variable grupo de deportes fue la única que apoyó parcialmente las hipótesis de investigación. Se puede decir que la motivación integral a la autosuperación y motivación integral del deportista a favor de los deportes de resistencia son características propias de dichas disciplinas deportivas en las que las exigencias competitivas y del entrenamiento pueden desarrollar en el deportista conductas autodeterminadas para trazar metas propias según cada deportista, este argumento se puede sostener a la luz de promedios superiores en la motivación intrínseca a la autosuperación.

Algunas de las aproximaciones en función a la variable de acuerdo con el grupo o modalidad deportiva han sido trabajadas en la comparación de deportes individuales versus deportes de conjunto (Moreno-Murcia et al., 2007) encontrando que en los deportes colectivos existe una mayor orientación al ego y una mayor percepción de un clima ego, mientras que en los deportes individuales es mayor la orientación a la tarea; así, estos resultados pueden coincidir y entender por qué en deportes de resistencia y en los que las condiciones sea el carácter individual parece correlacionarse que a mayor autodeterminación mayor orientación a la tarea, datos que son consistentes con otro estudio (Hanrahan y Biddle, 2002) donde se evidenciaron mayores niveles de motivación intrínseca o autodeterminación en deportistas practicantes de atletismo en comparación a deportistas de otras modalidades.

En cuanto al género, los resultados de la investigación no establecieron diferencias entre varones y mujeres, estos datos confirman previos hallazgos en esta misma dirección (Martín-Albo et al., 2007), en evaluaciones de motivación al logro tampoco se han encontrado diferencias entre varones y mujeres deportistas (Hanrahan y Biddle, 2002). Por otro lado, los promedios observados en varones, comparados con las mujeres, han señalado que los hombres han obtenido puntajes superiores (Balaguer et al., 2007; Moreno-Murcia, Cervelló-Gimeno y González-Cutre Coll, 2007; Núñez et al., 2006), lo que puede deberse a que el género masculino destina su búsqueda a la competitividad y, a través de ella, la recompensa. Asimismo, esta situación hace que las mujeres obtengan puntuaciones altas en perfiles autodeterminados (Moreno-Murcia et al., 2007). En esta misma dirección, un estudio previo (AguirreLoaiza y González 2014) encontró que en deportistas universitarios los varones presentaban diferencias significativas en comparación con las mujeres en factores de motivación extrínseca y amotivación y parece ser explicado por condiciones de competencia que parecen regular la conducta de los varones en brusquedad de recompensas ambientales. Aunque, si bien los datos no ofrecen la discriminación entre género, la discusión en cuanto a la característica de género sigue abierta y a la espera de nuevas contribuciones.

Ante el nivel competitivo y la edad deportiva, los estudios han considerado que la acción del deportista está regulada de manera voluntaria y por la práctica del deporte que desea (Navas, Soriano, Holgado y López, 2009). Esto cobra importancia si se observa detenidamente la relación del individuo con el contexto (Papaioannou, Marsh y Theodorakis, 2004), en esta lógica, puede pensarse que lo sugerido por otros autores frente a la importancia del entrenador en generar un clima motivacional adecuado en función a autodeterminación (López et al., 2011; Sánchez-Oliva et al., 2010, 2012). Aunque en condiciones previas a la competencia, la conducta está medida por la motivación extrínseca de regulación externa bajo determinantes pertinentes a la propia investigación (Guzmán, y Carratalá, 2006), los investigadores asumen que, de no efectuarse un predominio de la regulación externa, podría esperarse un mejor desempeño. 
A la vez, puede asumirse que el entrenador sea quién detente el rol de liderazgo y facilite experiencias positivas a sus deportistas, agregado a la amplia influencia que ejerce el entrenador sobre el compromiso deportivo del atleta (García Calvo, Leo, Martín y Sánchez-Miguel, 2008; Torregrosa, Sousa, Viladrich, Villamarín y Cruz 2008, Balaguer, Castillo y Duba, 2008) y propicie el clima en que la motivación del deportista esté encaminada a condiciones favorables tanto para sí mismo como para el equipo, por ejemplo, aquellos destinados a la tarea, y que según la literatura ha sugerido en diversos estudios (Moreno-Murcia et al., 2007).

Es importante advertir algunas de las limitaciones y, a su vez, sugerencias de cara a futuros trabajos, por ejemplo, contar con grupos normativos en la valoración de la EMD pertinentes al contexto colombiano, del mismo modo, pretender alcances de investigación a nivel experimental, además de contrastar otras variables como la condición de medallista-no medallista. Finalmente, es importante señalar que los diferentes

\section{REFERENCIAS BIBLIOGRÁFICAS}

Aguirre-Loaiza, H. y González, J. (2013). Factores motivacionales, variables deportivas y sociodemográficas en deportistas universitarios. Tesis Psicológica, 9(1), 130-145.

Almagro, B., Sáenz-López, P. y Moreno-Murcia, J. (2012). Perfiles motivacionales de deportistas adolescentes españoles. Revista de Psicología del Deporte, 21(2), 223-231.

Balaguer, G., Castillo, I. y Duda, J. (2007). Propiedades psicométricas de la escala de motivación deportiva en deportistas españoles. Revista Mexicana de Psicología, 24(2), 197-207.

Brière, N., Vallerand, R., Blais, N. y Pelletier, L. (1995). Dèveloppement et validation d'une mesure de motivation intrinsèque, extrinsèque et d'amotivation en contexte sportif: l'èchelle de motivation dans les sports (EMS). International Journal of Sport Psychology volumen (26), 465-489.

Chatzisarantis, N. L., Hagger, M. S., Biddle, S. J., Smith, B. y Wang, J. C., (2003). A meta-analysis of perceived locus of causality in exercise, sport and physical education contexts. Journal of Sport and Exercise Psychology, 25, 284-306.

Çetinkalp, Z. y Turksoy, A. (2011).Goal orientation and self-efficacy as predictors of male adolescent soccer tipos de motivación pueden coexistir y correlacionar positivamente (Standage y Treasure, 2002), por consiguiente, los anteriores resultados ofrecen apoyo al modelo jerárquico de la motivación (Vallerand, 1997 y 2000) y a la multidimensionalidad de la teoría de la autodeterminación (Deci y Ryan, 1985 y 2000). Únicamente se encontraron diferencias significativas en los factores motivación intrínseca a la autosuperación y motivación integral. No se encontraron diferencias significativas en los factores motivacionales en función del nivel competitivo, si bien los deportistas que se encuentran en el nivel mundial presentaron mayores puntajes. Se observó que el grupo de los menos experimentados arrojó promedios superiores en comparación con los demás grupos en los factores motivación extrínseca, motivación intrínseca al conocimiento, motivación intrínseca al cumplimiento, motivación integral-deportiva. De igual modo, cabe destacar que los deportistas que tienen más años en edad deportiva no arrojaron promedios superiores en comparación con los demás grupos con menor experiencia.

players' motivation to participate. Social Behavior and Personality, 39(7), 925-934.

Deci, E. y Ryan, R. (1985). Intrinsic motivation and self determination in human behavior. Nueva York: Plenum.

Dosil, J. (2008). Psicología de la actividad física y del deporte. Madrid: McGraw-Hill.

Durand, M. (1988). El niño y el deporte. Barcelona: Paidós.

García-Calvo, T., Leo, F., Martín, E. y Sánchez-Miguel, P.A. (2008). El compromiso deportivo y su relación con factores disposicionales y situacionales contextuales de la motivación. Revista Internacional de Ciencias del Deporte, 12(4), 45-58.

García-Calvo, T., Sánchez, P., Leo, F., Sánchez, D. y Amado, D. (2011). Incidencia de la Teoría de Autodeterminación sobre la persistencia deportiva. Revista Internacional de Ciencias del Deporte, 25(7), 266-276.

González, O., Salguero, A. y Márquez, S. (2011). Autodeterminación y adherencia al ejercicio: estado de la cuestión. RICYDE. Revista Internacional de Ciencias del Deporte, 7(25), 287-304.

Guzmán, J. y Carratalá, V. (2006). Mediadores psicológicos y motivación deportiva en judocas españoles. Revista Internacional de Ciencias del Deporte, 5(2), 1-11. 
Guzmán, J., Carratalá, E., García-Ferriol, A. y Carratalá, V. (2006). Propiedades psicométricas de una escala de motivación deportiva. Motricidad. European Journal of Human Movement, 16, 85-98.

Guzmán-Luján, J., García-Ferriol, A. y Cervelló-Gimeno, E. (2005). Percepción de competencia de las jugadoras y de criterios de éxito del entrenador como predictores de la orientación de metas en balonmano de base. Revista de Psicología del Deporte, 14(1), 7-19.

Hanrahan, S. y Biddle, S. (2002). Measurement of achievement orientations: psychometric measures, gender, and sport differences. European Journal of Sport Science, 2(5), 1-12.

Hernández, H., Vasconcelos-Raposo, J., Lázaro, J. y Dosil, J. (2004). Validación y aplicación de modelos teóricos de motivación en el contexto de la educación física. Cuadernos de Psicología del Deporte, 4(1-2), 67-89.

Kerlinger, F. (1988). Investigación del comportamiento. México: McGraw-Hill

Lavallee, D., Kremer, J., Moran, A. y Williams, M. (2004). Sport Psychology. Palgrave: MacMillan.

López, J. (2000). Estandarización de la escala de motivación en el deporte-EMD de Brière, Vallerand, Blais y Pelletier en deportistas mexicanos. Revista de Motricidad, 6, 67-93.

López, J., Balaguer, I., Castillo, I. y Tristán, J. (2011). Clima motivacional percibido, motivación autodeterminada y autoestima en jóvenes deportistas mexicanos. Revista de Psicología del Deporte, 20, 209-222.

Lonsdale, C. Hodge, K., Hargreaves, E. y Ng, J. (2014). Comparing sport motivation scales: a response to Pelletier et al. Psychology of Sports and Exercise, 15(5), 446-452.

Martens, M. y Webber, S. (2002). Psychometric properties of the Sport Motivation Scale: An evaluation with college varsity athletes from the U.S. Journal of Sport $y$ Exercise Psychology, 24, 254-270.

Martín-Albo, J., Núñez, J., Navarro, J., Leite, M., Almirón, M. y Glavinich, N. (2007). Propiedades psicométricas de la versión española de la Escala de Motivación Deportiva en Paraguay. Revista Mexicana de Psicología, 24(1), 43-52.

Moreno-Múrcia, J., Blanco, M., Galindo, C., Villodre, N. y González-Cutre, D. (2007). Efeitos do gênero, a idade e a frequência de prátican amotivação e o desfrute do exercício físico. Fitness y Performance Journal, 6(3), 140-146.

Moreno-Murcia, J., Cervelló-Gimeno, E. y González-Cutre, D. (2007). Analizando la motivación en el deporte: un estudio a través de la teoría de la autodeterminación. Apuntes de Psicología, 25(1), 35-51.
Navas, L., Soriano, J. y Holgado F. P. (2006).Orientaciones de meta en las clases de educación física: un análisis centrado en la educación secundaria obligatoria. Revista de Psicología del Deporte, 15(2), 167-181.

Núñez, J., Martín-Albo, J. y Navarro, J. (2007). Propiedades psicométricas de la versión española de la escala de motivación deportiva. Revista de Psicología del Deporte, 16(2), 211-223.

Núñez, J., Martín-Albo, J., Navarro, J. y González, V. (2006). Preliminary validation of a Spanish versión of the Sport Motivation Scale. Perceptual and Motor Skills, 102, 919-930.

Núñez, J., Martín-Albo, J., Paredes, A., Rodríguez, 0. y Chipana, N. (2011). The mediating role of perceived competence: testing a motivational sequence in university students. Universitas Psychologica, 10(3), 669-680.

Pelletier, L., Rocchi, M., Vallerand, R., Deci, E. y Ryan, R. (2013). Validation of the revised sport motivation scale (SMS-II). Psychology of Sport and Exercise, 14(3), 329-341.

Papaioannou, A., Marsh, H. y Theodorakis, Y. (2004). A multilevel approach to motivational climate in Physical Education and sport settings: An individual or group level construct? Journal of Sport and Exercise Psychology, 26, 90-118.

Reeve, J. (2010). Motivación y emoción. México: McGrawHill.

Rodríguez, M. y Lozada, E. (2007). Validación de la escala de motivación deportiva en Colombia. Revista Psilabas, $2,15-32$.

Ryan, R. y Deci, E. (2000). Self-determination theory and the facilitation of intrinsic motivation, social development and well-being. American Psychologist, 55(1), 68-78.

Sánchez-Oliva, D., Leo, F., Sánchez, P., Amado, D. y GarcíaCalvo, T. (2010). Relación entre los motivos de práctica y los comportamientos de deportividad en jóvenes jugadores de balonmano. E-balonmano.com: Revista de Ciencias del Deporte, 6 (3), 111-122.

Sánchez-Oliva, D., Leo, F., Sánchez, P., Amado, D. y GarcíaCalvo, T. (2012). Antecedentes motivacionales de los comportamientos prosociales y antisociales en el contexto deportivo. Revista Internacional de Medicina y Ciencias de la Actividad Física y el Deporte, 12(46), 253-270.

Standage, M., Duda, J. y Ntoumanis, N. (2005). A test of selfdetermination theory in school physical education. British Journal of Educational Psychology, 75(3), 411433. 
Torregosa, M., Sousa, C., Viladrich, C., Villamarín, F. y Cruz, J. (2008) El clima motivacional y el estilo de comunicación del entrenador como predictores del compromiso en futbolistas jóvenes. Psicothema, 20 (2), 254-259.

Vallerand, R. (1997). Toward a hierarchical model of intrinsic and extrinsic motivation. En M. Zanna (ed.), Advances in experimental social psychology, 29, 271360.
Vallerand, R. (2000). Deci and Ryan's Self-Determination theory: A view from the Hirearchical Model of Intrinsic and Extrinsic Motivation. Psychological Inquiry, 11(4), 312-319.

Vallerand, R. y Losier, G. (1999). An Integrative Analysis of Intrinsic and Extrinsic Motivation in Sport. Journal of Applied Sport Psychology, 11, 142-96.

Weinberg, R. (2009). Motivation. En B. W. Brewer (ed.), Handbook of sports medicine and science (pp. 7-17). Chennai: Wiley-Blackwell. 